\title{
On the Energy Spectrum and Ground-State Properties of the Decorated Heisenberg Spin Tube
}

\author{
V.O. CheranovskiI ${ }^{a, *}$ And E.V. Ezerskaya ${ }^{b}$ \\ ${ }^{a}$ Karazin Kharkov National University, Dept. of Chemistry, 61077, Svoboda sq. 4, Kharkov, Ukraine \\ ${ }^{b}$ Karazin Kharkov National University, Dept. of Physics, 61077, Svoboda sq. 4, Kharkov, Ukraine \\ The ground-state properties of decorated Heisenberg spin tubes with nearest- and next-nearest-neighbor \\ antiferromagnetic exchange interactions has been studied using perturbation theory and exact diagonalization \\ technique. The possibility of quantum phase transitions mediated by next-nearest neighbor interactions for these \\ tubes is shown. \\ PACS numbers: $75.10 . \mathrm{Jm}$
}

\section{Introduction}

We studied the energy spectrum and ground state spin densities distribution of spin tubes formed by folding of stripe fragment of rectangular spin $s_{1}$ lattice decorated by $s_{2}$ spins. These tubes have three-site unit cell and can be treated as a collection of $L$ cyclic fragments formed by $n$ unit cells (Fig. 1).

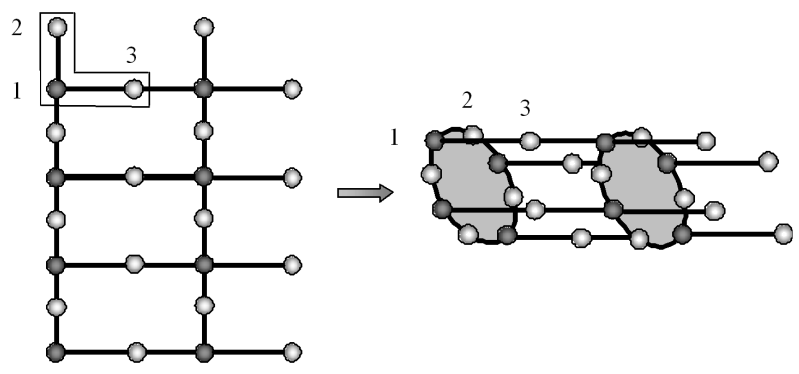

Fig. 1. Fragment of decorated spin tube formed by 8 unit cells $(n=4, L=2)$. The numeration of site spins inside of one unit cell is shown.

\section{The model}

In order to study the effect of next-nearest neighbor interactions (geometric frustration) on the tube energy spectrum we used the following simplified Heisenberg spin Hamiltonian:

$$
\boldsymbol{H}=\sum_{l=1}^{L}\left(\boldsymbol{H}_{l}+\alpha \boldsymbol{V}_{l, l+1}\right),
$$

with

$$
\begin{aligned}
& \boldsymbol{V}_{l, l+1}=\sum_{k=1}^{n} \boldsymbol{S}_{1, k, l+1} \boldsymbol{S}_{3, k, l}, \\
& \boldsymbol{H}_{l}=\sum_{k=1}^{n}\left[\boldsymbol{S}_{1, k, l}\left(\boldsymbol{S}_{2, k, l}+\boldsymbol{S}_{2, k-1, l}+\beta \boldsymbol{S}_{3, k, l}\right)\right. \\
& \left.\quad+\eta \sum_{i=1}^{2} \boldsymbol{S}_{i, k, l} \boldsymbol{S}_{i, k+1, l}\right], \quad \boldsymbol{S}_{i, n+1, l}=\boldsymbol{S}_{i, 1, l}, i=1,2 .
\end{aligned}
$$

* corresponding author; e-mail: cheranovskii@univer.kharkov.ua
Here $\boldsymbol{S}_{i, k, m}$ is the spin operator located on $i$-th lattice site of the unit cell labeled by index $k, m$ is a number of the corresponding cyclic fragment; $i=1$ corresponds to the site spin $s_{1}$ and $i=2,3$ correspond to the site spins $s_{2}$. The parameter $\alpha$ represents a relative strength of the nearest-neighbor interaction between the spins from the bonds connecting neighboring cyclic fragments of the tube, while the parameters $\beta$ and $\eta$ stands for nearest-neighbor and next-nearest-neighbor interactions between the pairs of spins $\left(\boldsymbol{S}_{1, k, l}, \boldsymbol{S}_{2, k, l}\right)$ and $\left(\boldsymbol{S}_{1, k, l}\right.$, $\left.\boldsymbol{S}_{2, k, l}\right)$, respectively, inside the cyclic fragment with number $l$ (Fig. 1).

At $\alpha=1$ the Hamiltonian (1) describes the tubular fragment of magnetic sublattice of some real quasi-two-dimensional bimetallic ferrimagnets. For example, for complex salt $\left[\mathrm{Ni}(\mathrm{pn})_{2}\right]_{2}\left[\mathrm{Fe}(\mathrm{CN})_{6}\right] \mathrm{ClO}_{4} \cdot 2 \mathrm{H}_{2} \mathrm{O}(\mathrm{pn}-1,2-$ -propanediamine) all the spins $S_{1, m, l}$ correspond to ions $\mathrm{Fe}^{3+}$ with $s_{1}=1 / 2$ and all other spins correspond to ions $\mathrm{Ni}^{2+}$ with $s_{2}=1[1]$.

At $\eta=0$ our spin tubes have bipartite structure. Therefore, according to the extension of the Lieb-Mattis theorem [2, 3] the Hamiltonian (1) should have non-degenerate ground state with the total spin $S_{0}=$ $L n\left|2 s_{2}-s_{1}\right|$. At $s_{1}=s_{2}=1 / 2$ the exact ground state of (1) has a spin wave structure [4] (spin densities on neighboring lattice sites have opposite signs).

\section{Discussion}

It is known that the next-nearest neighbor interactions (frustration) may enhance the quantum spin fluctuation and suppress the magnetic order [5]. In other words, the increase of $\eta$ may lead to the decrease of $S_{0}$. In order to check this suggestion we performed exact diagonalization study of the lowest energy levels with the specified value of total spin $S$ for small tube fragments with $s_{1}=s_{2}=1 / 2$ and $n=3, L=2$ by means of the Davidson method [6]. The branching diagram technique was used for the construction of the Hamiltonian matrix elements in spin symmetry adopted basis. According to our numerical calculations the increase of $\eta$ leads to the jump in the value of $S_{0}$ for these fragments. For example, for $\alpha=\beta=0.5$ we found the $S_{0}=3 \rightarrow S_{0}=0$ at $\eta_{\mathrm{c}} \approx 0.474$ for the second tube fragment (Fig. 2). 


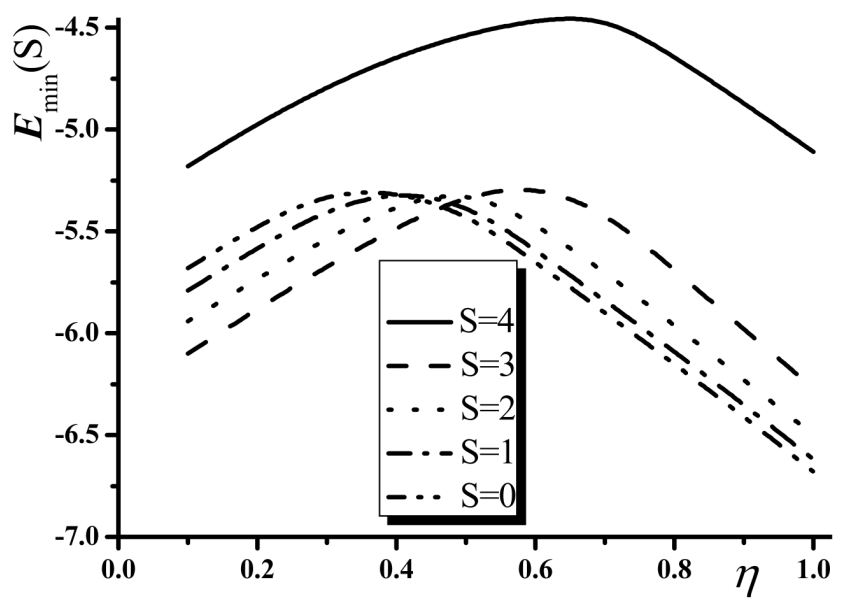

Fig. 2. The lowest energy states with different values of total spin $S$ of decorated spin tube fragment $\left(s_{1}=s_{2}=1 / 2, L=3, n=2\right)$.

In order to study the effect of frustrations on the ground state of infinite spin tubes, let us consider the tube formed by weakly interacting cyclic fragments $(0<\alpha \ll 1)$. Let us suppose that the isolated fragment of the tube has non-degenerate ground state $\phi(\tilde{S}, m)$ with the ground state spin $\tilde{S}$ and $z$-projection of this spin $m\left(\tilde{S}=n\left|2 s_{2}-s_{1}\right|\right.$ for tubes with $\left.\eta=0\right)$. In first order of perturbation theory (PT) in parameter $\alpha$ the lowest energy states of the tube $\Psi_{\{m\}}(M)$ have the form of direct product of functions $\phi(\tilde{S}, m)$ :

$$
\begin{aligned}
& \Psi_{\{m\}}(M)=\varphi\left(\tilde{S}, m_{1}\right) \times \varphi\left(\tilde{S}, m_{2}\right) \times \ldots \\
& \quad \times \varphi\left(\tilde{S}, m_{L}\right), \quad M=\sum_{i=1}^{L} m_{i} .
\end{aligned}
$$

The matrix elements of the Hamiltonian (1) in subspace spanned by functions $\Psi_{\{m\}}(M)$ can be evaluated by means of the Wigner-Eckart (replacement) theorem. As the result it can be shown that the low-energy states of the tube are described by effective Heisenberg Hamiltonian of uniform spin- $\tilde{S}$ chain

$$
\boldsymbol{H}_{\mathrm{eff}}=J_{\mathrm{eff}} \sum_{l=1}^{L} \tilde{\boldsymbol{S}}_{l} \tilde{\boldsymbol{S}}_{l+1}+L \tilde{\varepsilon}_{0}, \quad J_{\mathrm{eff}}=\frac{n \rho_{1} \rho_{3}}{\tilde{S}^{2}} \alpha,
$$

where $\rho_{i}=\left\langle\varphi(\tilde{S}, \tilde{S})\left|\boldsymbol{S}_{i, m, l}^{z}\right| \varphi(\tilde{S}, \tilde{S})\right\rangle, \tilde{\varepsilon}_{0}$ is the ground state energy of cyclic fragment.

We performed the exact diagonalization study of the energy spectra of cyclic tube fragments with $n=2-4$, and $s_{1}=1 / 2, s_{2}=1 / 2,1$. According to our calculations, at small values of parameter $\eta$ the exact ground state of cyclic fragment is non-degenerate and corresponds to the spin $\tilde{S}=n\left(2 s_{2}-s_{1}\right)$. The ground state spin densities $\rho_{1}, \rho_{3}$ have opposite signs. As the result, the effective coupling is ferromagnetic $\left(J_{\text {eff }}<0\right)$.
Therefore the Hamiltonian (2) has the ground state spin $S_{0}=\operatorname{Ln}\left(2 s_{2}-s_{1}\right)$ and gapless excitation spectrum at $L \rightarrow \infty$.

At $\alpha \ll 1$ the lowest excited state of the tube with $S \geq \tilde{S}$ has the form of linear combination

$$
\begin{aligned}
& \Psi_{1}=\sum_{k=1}^{L} a_{k} \Phi_{k}(M) \\
& \Phi_{k}(M)=\varphi\left(\tilde{S}, m_{1}\right) \times \ldots \times \phi\left(S, m_{k}\right) \times \ldots \\
& \quad \times \varphi\left(\tilde{S}, m_{L}\right)
\end{aligned}
$$

where $\phi\left(S, m_{k}\right)$ is the lowest excited state of cyclic fragment.

This state is separated by finite energy gap from the ground state due to the non-degeneracy of the fragment ground state. Therefore for weak coupling between cyclic fragments there is finite energy gap between the excitations with $S \geq S_{0}$ and the ground state of the tube. Due to this gap at low temperatures the field dependence of tube magnetization should have an intermediate plateau which corresponds to $z$-projection of total spin per unit cell $M=2 s_{2}-s_{1}$.

\section{Conclusions}

According to our numerical calculations the increase of $\eta$ leads to the decrease of the value of $\tilde{S}$. For example, at $\eta=0$ the cyclic tube fragment with $n=3$ and $s_{1}=1 / 2, s_{2}=1$ has the ground state $\operatorname{spin} \tilde{S}=9 / 2$. It decreases to the value $\tilde{S}=7 / 2$ at $\eta_{\mathrm{c}} \approx 0.2493$. When $\eta$ exceeds the value $\eta_{\mathrm{c}}^{*} \approx 0.2516$, the fragment ground state spin exhibits jump to the minimal value $\tilde{S}=1 / 2$. For spin tube with $n=4$ we found stepwise decrease of the ground state spin to zero value at $\eta_{\mathrm{c}} \approx 0.2315$. In first PT order in $\alpha$ the lowest energy states of infinite tube are created from the direct products of the lowest energy states of cyclic fragments. On the other hand, for both cyclic fragments at $\eta \leq \eta_{\mathrm{c}}$ our numerical calculations give ferromagnetic coupling constant $J_{\text {eff }}$ in (2). This means that the decrease of the value of $\tilde{S}$ leads to macroscopic jump in the value of the ground state spin of infinite tubes considered. In other words, decorated spin tubes formed by weakly interacting cyclic fragments may demonstrate quantum phase transitions mediated by geometrical frustration.

\section{References}

[1] M. Ohba, H. Okawa, N. Fukita, Y. Hashimoto, J. Am. Chem. Soc. 119, 1011 (1997).

[2] E.H. Lieb, D.C. Mattis, J. Math. Phys. 3, 749 (1962).

[3] G.-S. Tian, Phys. Rev. B 56, 5355 (1997).

[4] A.A. Ovchinnikov, V.O. Cheranovskii, Theor. Exp. Chem. 16, 119 (1980).

[5] T. Tonegawa, K. Okamoto, K. Okunishi, K. Nomura, Physica B 346-347, 50 (2004).

[6] E.R. Davidson, J. Comp. Phys. 17, 87 (1975). 